Europhys. Lett., 3 (5), pp. 593-600 (1987)

\title{
Non-Markovian Description of the Hedvall Effect $(*)$.
}

\author{
U. SEIFERT and S. DieTRICH \\ Sektion Physik der Universität München - Theresienstr. 37, D-8000 München 2, \\ Federal Republic of Germany
}

(received 8 September 1986; accepted in final form 14 November 1986)

PACS. 64.60. - General studies of phase transitions.

PACS. 75.40D - Ising and other classical spin models.

PACS. 82.65. - Surface processes.

\begin{abstract}
Activated processes at surfaces-like desorption or oxidation-exhibit thermal anomalies at phase transitions of the underlying substrate. Inter alia, such singularities in the case of a continuous transition are caused by the critical slowing down in the substrate, which leads to pronounced memory effects in the viscosity coefficient. Therefore, we apply a nonMarkovian analog of Kramers' classical rate theory. As a result, the anomalies can be expressed in terms of critical exponents associated with the critical surface behaviour.
\end{abstract}

\section{Introduction.}

If a solid undergoes a second-order phase transition at the temperature $T=T_{\mathrm{c}}$, thermally activated processes at its surface may exhibit anomalies in their reaction rate $r(T)$. This socalled Hedvall effect $[1,2]$ has been discovered in 1934. Meanwhile the experimental evidence includes as different processes as catalytic reactions [3,4], oxidation [5-8], sublimation [9] and desorption [10]. Outside the critical region, i.e. for $|\tau| \simeq 1$ with $\tau=\left(T-T_{\mathrm{c}}\right) / T_{\mathrm{c}}$, the reaction rate follows the usual Arrhenius law $r(T)=k \exp \left[-Q / k_{\mathrm{B}} T\right]$. For $|\tau| \ll 1$, however, either the prefactor $k$ (Hedvall effect I) or the activation energy $Q$ (Hedvall effect II) or both become temperature dependent. Consequently, the Arrhenius plot $\ln r v s . T^{-1}$ shows cusp-like singularities or a break in slope at $T_{\mathrm{c}}$ -

The starting-point for a theoretical understanding of these experimental facts is based on the observation, that such thermal singularities can occur only if the activated process is coupled to the appropriate order parameter $(\mathrm{OP})$ of the phase transition. This coupling shows up in the activation energy $Q$ as well as in the noise driving the process. As a basic hypothesis, the latter contribution to the singularity close to $T_{\mathrm{c}}$-originates from that part of the noise which stems from the critical OP-fluctuations near the surface.

Studies along this line of arguments were started by SUHL and co-workers [11-14] about

(*) This work has been supported by the Bundesministerium für Forschung und Technologie under Grant No. 03WA1LMU/5. 
ten years ago. Combining Kramers' theory of activated processes [15] with the Kubo formalism they expressed the reaction rate in terms of correlation functions describing the relevant degrees of freedom of the substrate. The basic assumption of the Kramers approach is a clear separation of the time-scales involved. In order to treat the substrate as a heat bath its fluctuations must be much faster than a typical reaction time. This condition, however, breaks down close to $T_{\mathrm{c}}$ due to the critical slowing down.

We, therefore, employ a generalized Kramers approach, which renounces this limiting condition. Our work presented in this letter takes advantage of studies on non-Markovian rate processes [16-18]. We show that in this case the reaction rate is determined by different features of the correlation functions compared to the classical Kramers approach.

Since the reaction takes place at the surface where the critical behaviour is different from that in the bulk, one needs the relevant dynamical correlation functions for systems with surfaces. These have recently been evaluated systematically with field-theoretical methods of the renormalization group [19-21]. With these results we find that the singularity of the rate is governed by critical surface exponents. This important feature was missed in a recent letter by BORMAN et al. [22] who evaluated the Markovian Kramers-Suhl expression by treating the substrate within mean-field theory as a pure bulk system.

In sect. 2 we present the generalized Kramers approach. In particular, we discuss the reaction rate for friction with arbitrarily slow-decaying memory. In sect. 3 we apply these results to a specific model for the Hedvall effect. Our conclusions are summarized in sect. 4.

\section{The generalized Kramers approach.}

Within the Kramers model [15] the activated process is described by the movement of a reaction particle in a potential $V(z)$ (fig. 1$)$ along the reaction co-ordinate $z$. We will describe this model for a desorption process in order to have a clear terminology. In that case the reaction particle is the adparticle itself and $z$ corresponds to the co-ordinate perpendicular to the surface $(z=0)$. The metastable state at $z_{A}$ corresponds to the adsorption well. Desorption takes place if the particle is thermally activated through couplings to the substrate. It crosses the desorption barrier at $z_{B}$ escaping to infinity. The reaction rate $r$ is determined by considering the flux over the barrier at $z_{B}$. In our case the driving noise is

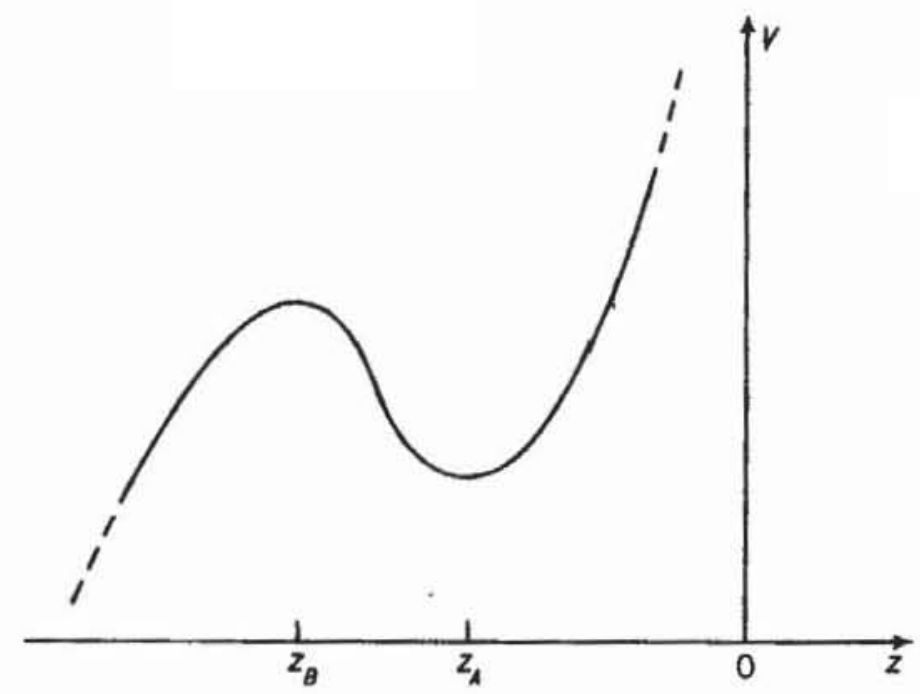

Fig. 1. - Potential energy $V(z)$ of the adparticle. The substrate fills the half-space $z>0 . z_{A}$ and $z_{B}$ correspond to the adsorption well and the desorption barrier, respectively. 
correlated within a finite correlation time $t_{\mathrm{c}}$ which turns out to diverge for $T \rightarrow T_{\mathrm{c}}$ (see below). Therefore, we use a generalized Langevin equation for the moving adparticle of mass $M$ [16-18]:

$$
\ddot{z}(t)+\int_{0}^{1} \mathrm{~d} s \eta(t-s) \dot{z}(s)+M^{-1} \partial_{2} V=M^{-1} \zeta(t) .
$$

The memory effects of the stochastic forces $\zeta(t)$ show up in the friction kernel $\eta(t)$. Both quantities are connected by the fluctuation-dissipation theorem

$$
\eta(t)=\left(M k_{\mathrm{B}} T\right)^{-1}\langle\zeta(0) \zeta(t)\rangle .
$$

In the case of white noise, i.e. $\eta(t)=2 \eta_{K} \delta(t)$, KRAMERS [15] solved the corresponding Fokker-Plank equation approximately. He found for the rate $r$

$$
r=k \exp \left[-Q / k_{\mathrm{B}} T\right],
$$

where

$$
Q=V\left(z_{B}\right)-V\left(z_{A}\right) .
$$

The attempt frequency $k$ is given by

$$
k=\left(\omega_{A} / 2 \pi \omega_{B}\right) \lambda
$$

with

$$
\lambda=\lambda_{\mathrm{K}}=\left(\left(\eta_{\mathrm{K}}^{2}+4 \omega_{B}^{2}\right)^{1 / 2}-\eta_{\mathrm{K}}\right) / 2
$$

and the frequencies $\omega_{A, B}^{2}=V^{\prime \prime}\left(z_{A, B}\right) / M$.

Equation (4) is valid for intermediate and high friction $\left(\eta_{\mathrm{K}} \rightarrow \omega_{B}\right)$. In this case the reaction is determined by position diffusion over $z_{B}$. The formal limit $\eta_{K} \rightarrow 0$ in eq. (4) leads to the attempt frequency $k=\omega_{A} / 2 \pi$ which corresponds to the value obtained within absolute ratetheory. For small $\eta_{\mathrm{K}}$ energy diffusion is the rate determining step. In that case Kramers found $k=\eta_{\mathrm{K}} Q / k_{\mathrm{B}} T$.

Near $T_{\mathrm{c}}$, however, the OP-fluctuations in the substrate decay slowly in time. Therefore, we need the rate following from the non-Markovian eq. (1) with arbitrary friction kernel $\eta(t)$. Various papers have been concerned with this problem. We refer to them by just quoting the remarkably simple approximate solution [16-18]: eqs. (3) are still valid, but with $\lambda$ given by the non-Markovian value $\lambda_{\mathrm{nM}}$ which is the implicit solution of

$$
\omega_{B}^{2} / \lambda_{\mathrm{nM}}-\lambda_{\mathrm{nM}}=\hat{\eta}\left(\lambda_{\mathrm{nM}}\right) .
$$

$\hat{\eta}(\lambda)$ denotes the Laplace transform of the friction kernel:

$$
\hat{\eta}(\lambda)=\int_{0}^{\infty} \mathrm{d} t \exp [-\lambda t] \eta(t)=\pi^{-1} \int_{0}^{\infty} \mathrm{d} \omega \lambda\left(\lambda^{2}+\omega^{2}\right)^{-1} \tilde{\eta}(\omega),
$$

where we introduced the Fourier transform

$$
\bar{\eta}(\omega)=\int_{-\infty}^{+\infty} \mathrm{d} t \exp [i \omega t] \eta(t)
$$


of the friction kernel $\eta(t)=\eta(-t)$. As above, this solution is reliable for sufficient high friction which guarantees that the reaction dynamics is determined by position diffusion over $z_{B}$. Note that Kramers' result (eq. (4)) is recovered by inserting $\eta(t)=2 \eta_{\mathrm{K}} \delta(t)$ into eqs. (5).

Before applying eqs. (5) to a specific model for the Hedvall effect, let us discuss the consequences of this result in general terms. As stated in sect. 1 the time dependence of the noise we are concerned with is governed by the critical dynamics of the OP. Therefore, the correlation time $t_{\mathrm{c}}$ diverges by approaching $T_{\mathrm{c}}: t_{\mathrm{c}}=\lambda_{0}|\tau|^{-v z}[19] . z(\simeq 2.01$ for a nonconserved $\mathrm{OP})$ is the dynamical critical exponent and $\nu(\simeq 0.63)$ characterizes the diverging correlation length: $\xi=\xi_{0}|\tau|^{-\nu}$. $\lambda_{0}$ and $\xi_{0}$ are microscopic time- and length-scales. The scaling property of the OP-correlation function leads to a scaling law for the long-time behaviour of the friction kernel:

$$
\eta(t, \tau)= \begin{cases}A t^{-\mu} f\left(t / t_{\mathrm{c}}\right), & \text { for } t \gg \lambda_{0}, \\ \eta_{0}\left(t, t_{\mathrm{c}}\right), & \text { for } t \leqslant \lambda_{0} .\end{cases}
$$

Since at criticality $\eta(t)$ decays like a power law, one has

$$
f\left(t / t_{\mathrm{c}}\right)=1-a\left(t / t_{\mathrm{c}}\right)^{\phi+} \ldots, \quad\left(t / t_{\mathrm{c}}\right) \ll 1 .
$$

For short times, the leading temperature dependence of $\eta$ is

$$
\eta_{0}\left(t, t_{\mathrm{c}}\right)=\eta_{0}(t)+\bar{\eta}_{0}(t) t_{\mathrm{c}}^{-\sigma}+\ldots .
$$

$\mu, \phi$ and $\sigma$ are determined by the OP-dynamics (see below). From eqs. (3c), (5), (6) one obtains

$$
k(\tau)=k(0)+\bar{k}|\tau|^{x},
$$

where

$$
x=x_{\mathrm{nM}}=v z \min (\sigma, \phi)>0 \quad \text { and } \bar{k}>0 .
$$

This constitutes our first general result. Within our basic hypothesis (see sect. 1), eqs. (7) express the thermal singularity of the attempt frequency $k$ in terms of critical exponents of the OP.

As a limiting case, our theory allows to recover the classical Kramers-Suhl approach as applied by BORMAN et al. [22]. These authors consider the time-scale, on which the stochastic forces vary, to be much shorter than that of the deterministic motion of the adparticle. Then the friction kernel $\eta(t)$ looses its memory only retaining its integral strength:

$$
\eta_{K}=\int_{0}^{\infty} \eta(t) \mathrm{d} t=\bar{\eta}(0) / 2 .
$$

For $\mu>1$, eqs. (3c), (4), (6) yield in eq. (7a)

$$
x=x_{\mathrm{K}}=v z \min (\mu-1, \phi)>0 .
$$


For a very slowly decaying friction kernel, i.e. $0<\mu \leqslant 1, k_{\mathrm{K}}(\tau) \propto \tau^{(1-\mu) v z}$ vanishes at $T_{\mathrm{c}}$. However, as eqs. ( $7 a, b)$ show, memory effects yield $k_{\mathrm{nM}}(0)>0$ even for $0<\mu \leqslant 1$. We want to point out that this Markovian description breaks down at $T_{\mathrm{c}}$, because the clear separation of time-scales is lost due to the critical slowing down.

\section{A model for the magnetically induced Hedvall effect.}

The friction kernel $\eta(t)$ and the potential $V(z)$ are the only input entering the expression of the rate (eqs. (3), (5)). Within the Kramers approach both are phenomenological quantities and have to be specified from a microscopic theory. We now consider a crude model, which nevertheless is supposed to contain the important features. Our aim is to focus on the vicinity of $T_{\mathrm{c}}$ and thus to study the deviations of the rate from its noncritical value, which is caused by the coupling of the adparticle to the magnetic degrees of freedom of the substrate. This leads to consider the following interaction between the spin $S$ of the adparticle and the OP $s\left(r^{\prime}, t\right)$; $s\left(r^{\prime}, t\right)$ denotes the Ising spin density of the magnetic moments of the substrate with the easy axis $l$ :

$$
H_{\text {int }}(z, t)=S \cdot l \int \mathrm{d} \boldsymbol{r}^{\prime} J\left(\boldsymbol{r}^{\prime}-\boldsymbol{r}\right) s\left(\boldsymbol{r}^{\prime}, t\right) .
$$

The integration runs over the half-space $r^{\prime}=\left(x^{\prime}, z>0\right), x^{\prime}$ denotes the two-dimensional vector parallel to the surface at $z=0 . H_{\text {int }}$ depends on the position $r=(0, z)$ of the adparticle. Its motion is confined perpendicular to the surface.

In lowest-order perturbation theory in $J, S$ is constant and the dynamics of the OP is not affected by that of the adparticle. The critical OP-dynamics is governed by its own Langevin equation [19-21]

$$
\S\left(\boldsymbol{r}^{\prime}, t\right)=-\lambda_{0}^{-1}\left(\delta H_{s} / \delta s\right)+\left(\boldsymbol{r}^{\prime}, t\right)
$$

with Gaussian white noise $\left\langle f\left(\boldsymbol{r}^{\prime}, 0\right) f\left(\boldsymbol{r}^{\prime \prime}, t\right)\right\rangle=2 \lambda_{0}^{-1} \delta\left(\boldsymbol{r}^{\prime}-\boldsymbol{r}^{\prime \prime}\right) \delta(t) . H_{s}$ is the usual LandauGinzburg Hamiltonian for semi-infinite media with a continuous phase transition:

$$
H_{s}=\int \mathrm{d} \boldsymbol{r}^{\prime}\left(\frac{1}{2}(\nabla s)^{2}+(\tau / 2) s^{2}+(g / 4 !) s^{4}+\delta(z) c s^{2}\right) .
$$

$g>0$ stabilizes $H_{s}$ for $\tau<0$. $c>0$ takes into account that the magnetic moments at the surface have fewer neighbours. For $\tau \rightarrow 0$ eqs. (9) give the full description of the critical slowing down. Due to eq. (8) this dynamics imposes a time-dependent force $F(z, t)=-\partial_{z} H_{\text {int }}(z, t)$ on the adparticle which provides the noise and contributes to the systematic potential $V(z)$ (see eq. (1)).

In the first step we consider $V(z)=V_{0}(z)+V_{\text {int }}(z)$. $V_{0}(z)$ is that part of the potential energy of the adparticle, which originates from the nonmagnetic interaction with the substrate. We assume a typical and temperature-independent shape as in fig. 1. Specifying to a short-range exchange interaction, the magnetic part $V_{\text {int }}(z)$ follows from eq. (8) with $J_{0}\left(z^{\prime}-z\right)=\int d x^{\prime} J\left(r^{\prime}-r\right):$

$$
V_{\text {int }}(z)=\left\langle H_{\text {int }}(z, t)\right\rangle=S \cdot \boldsymbol{l} \int_{0}^{\infty} \mathrm{d} z^{\prime} J_{0}\left(z^{\prime}-z\right)\langle s(z, t)\rangle .
$$

Here and below averages $\langle\ldots\rangle$ are evaluated according to the stochastic dynamics of eqs. (9). The surface induces an inhomogeneous magnetization profile $\langle s(z)\rangle=m(z)$. For all $z$ 
within a slab of diverging thickness $\xi$, the temperature dependence of $m(z)$ is given by $m(z) \propto|\tau|^{\beta_{1}}$. We focus on the so-called ordinary transition for which the surface exponent $\beta_{1} \approx 0.82[23]$. Thus

$$
Q_{\text {int }}(\tau):=V_{\text {int }}\left(z_{B}\right)-V_{\text {int }}\left(z_{A}\right)=Q_{1}|\tau|^{\beta_{1}}, \quad \tau \rightarrow 0^{-} .
$$

The amplitude $Q_{1}$ may be positive or negative. This magnetic contribution to the activation energy $Q:=\left(V_{0}\left(z_{B}\right)-V_{0}\left(z_{A}\right)\right)+Q_{\text {int }}$ varies with temperature and vanishes above $T_{\mathrm{c}}$.

Since the extrema $z_{A, B}$ and the curvatures $\omega_{A, B}$ are given by the full potential $V(z)$, they are slightly changed:

$$
\omega_{A, B}(\tau)=\omega_{A, B}(0)+\bar{\omega}_{A, B}|\tau|^{\beta_{1}}, \quad \tau \rightarrow 0^{-} .
$$

In the second step we determine the friction kernel $\eta(t)$ using the fluctuation-dissipation theorem (eq. (2)). The stochastic forces on the adparticle follow from eq. (8): $\zeta(z, t)=-\partial_{z}\left(H_{\text {int }}(z, t)-V_{\text {int }}(z)\right)$. Contrary to the position-independent forces $\zeta(t)$ entering eqs. (1), (2), in principle this expression does depend on $z$. However, since the temperature dependence of $\left\langle\zeta(z, 0) \zeta\left(z^{\prime}, t\right)\right\rangle$ is the same for all relevant $z$ and $z^{\prime}$ [19-21], we may evaluate this correlation function at $z=z^{\prime}=0$. Since $J$ is short-ranged on the scale of $\xi$ this temperature dependence is captured correctly by setting $J\left(\boldsymbol{r}^{\prime}-\boldsymbol{r}\right)=J^{*} \delta^{\prime}\left(\boldsymbol{r}^{\prime}-\boldsymbol{r}\right)$. With eq. (8) this yields for eq. (2)

$$
\eta(t, \tau)=\left(M k_{\mathrm{B}} T\right)^{-1} J^{* 2}(S \cdot l)^{2} \gamma(t, \tau) .
$$

$\gamma(t, \tau)=\left\langle\left.\left.\partial_{z} s((0, z), 0)\right|_{z=0} \partial_{z} s((0, z), t)\right|_{z=0}\right\rangle_{c}$ is the cumulant of the autocorrelation function of the normal derivative of the surface spin density. Equation (12) qualifies our hypothesis that the noise and hence the friction is determined by critical surface correlation functions.

Recently, $\gamma$ has been analysed by field-theoretical techniques [20,21]. It fulfills the scaling property in eq. (6a) for $t \gg \lambda_{0}$ with $\mu=2 \beta_{1} / v z \simeq 1.30$.

The singular temperature dependence of the static two-point correlation function is the same for all distances $r \ll \xi$ and is given by that of the energy density, which corresponds to the formal limit $r \rightarrow 0$ [24]. From the occurrence of the critical slowing down we expect the corresponding behaviour for the time dependence of the autocorrelation function: Its singular temperature dependence is the same for all times $t \ll t_{\mathrm{c}}$ and is also given by that of the static energy density, which corresponds here to the formal limit $t \rightarrow 0$. Thus the exponents $\phi$ and $\sigma$ (eqs. (6)) are expected to be identical. This has been confirmed explicitly in the bulk [25]. For our case, the temperature dependence of $\gamma(0, \tau)$ has been evaluated in ref. [26] for $\tau>0$ :

$$
\gamma(0, \tau)=\gamma(0,0)\left(1-2 \tau(2-\alpha) /(1-\alpha)-\tau^{2}(2-\alpha) / \alpha+2 \tau^{2-\alpha / \alpha}(1-\alpha)\right),
$$

where $\alpha \simeq 0.11$ is the exponent of the specific heat. Thus one has $\phi=\sigma=1 / v z$ by means of eqs. (6) and $\tau \propto t_{\mathrm{c}}^{-1 / v z}$.

Collecting these results, we find for eqs. (7) in our model

$$
x_{\mathrm{K}}=0.37, \quad x_{\mathrm{nM}}=1 .
$$

(Strictly speaking for $\tau<0, x_{\mathrm{nM}}=\beta_{1}$, through eqs. (3c), (5), (11) if $\bar{\omega}_{A, B} \neq 0$.) Combining eqs. (3), (7), (10b), (13), we can state our final result for the singularity of the rate in the Arrhenius law:

$$
\ln r(\tau)=\ln r(0)+r^{ \pm}|\tau|^{p^{ \pm}}
$$




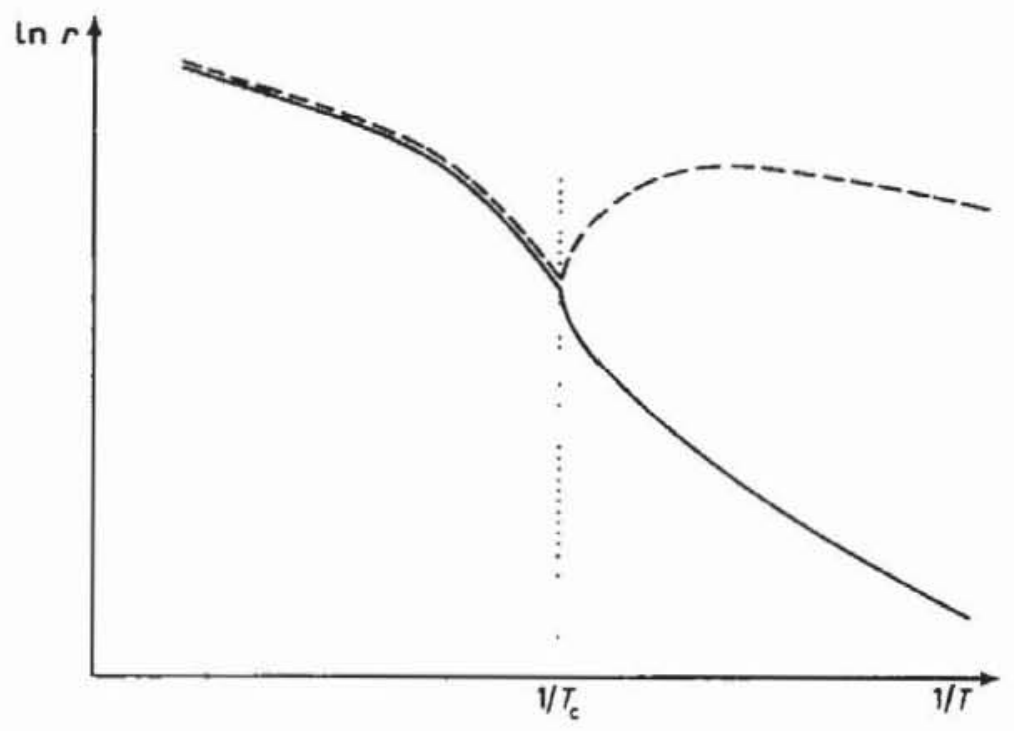

Fig. 2. - Arrhenius plot of the rate $r(T)$ for different signs of the amplitude $\bar{r}_{\mathrm{nM}}^{-}\left(\bar{r}_{\mathrm{nM}}^{-}>0\right.$ : dashed line, $\tilde{r}_{\mathrm{nM}}^{-}<0$ : solid line) in the case of intermediate and large friction (eq. (14)).

with $\bar{r}_{\mathrm{nM}}^{+}>0, \rho_{\mathrm{nM}}^{+}=x_{\mathrm{nM}}=1$ for $\tau>0$. For $\tau<0, \rho_{\mathrm{nM}}^{-}=\beta_{1} \approx 0.82\left(\tilde{r}_{\mathrm{nM}}^{-}=-Q_{1} / k_{\mathrm{B}} T\right.$ if $\left.\vec{\omega}_{A, B}=0\right)$. $\rho_{n \mathrm{M}}^{ \pm}$are static critical exponents. Therefore, these results apply also to a Heisenberg-like substrate with a slightly different numerical value for $\beta_{1}(\simeq 0.85)$.

For comparison we give the exponents for the Markovian Kramers case $\rho_{\mathrm{K}}^{+}=\rho_{K}=x_{K} \approx 0.37$. This last result corrects the values obtained by BoRMAN et al. [22].

The Arrhenius plots for the non-Markovian case are sketched in fig. 2 including constant noncritical contributions. Depending on the sign of $\bar{r}_{\mathrm{nM}}, \ln r$ shows a cusp pointing downward or a break in slope at $T_{\mathrm{c}}$.

Besides the ordinary transition considered here, other types of phase transitions can occur. At the so-called surface and special transition, the magnetic contribution to the activation energy for $T<T_{\mathrm{c}}$ shows a stronger singularity vanishing as $\left(T_{\mathrm{c}}-T\right)^{1 / 8}$ and $\left(T_{\mathrm{c}}-T\right)^{0.25}$, respectively [23]. A gadolinium substrate may exhibit such a behaviour [27].

\section{Summary.}

We presented a theory for the Hedvall effect which shows three essential characteristics different from previous approaches:

1) Memory effects require a non-Markovian description.

2) The relevant critical behaviour of the substrate is that of its surface.

3) Fluctuations are completely incorporated using renormalized instead of mean-field correlation functions.

We evaluated the singularity of the reaction rate at $T_{\mathrm{c}}$. Below $T_{\mathrm{c}}$, it is caused by the temperature-dependent magnetic contribution to the activation energy which vanishes at $T_{\mathrm{e}}$ as $\left(T_{\mathrm{c}}-T\right)^{0.8}$. Above $T_{\mathrm{c}}$, the singularity is determined by that of the attempt frequency which turns out to be a linear one.

A detailed comparison of our results with experimental data is rather difficult, since the temperature resolution of these experiments is insufficient in order to extract the exponent associated with the singularity. We find a qualitative agreement with, e.g., the desorption rate of hydrogen from nickel [10], which is reduced at $T=T_{\mathrm{c}}$ in accordance with fig. 2 .

On the contrary, other experiments indicate an enhanced rate at $T_{c}$, e.g. for the initial 
oxidation rate of iron [5]. Such a behaviour follows also from the Kramers approach if the friction is small and hence energy diffusion is the rate determining step. A non-Markovian theory for this case has not yet been developed if the memory kernel decays according to a power law as it occurs at $T_{\mathrm{c}}$. If one ignores this complication and uses a Markovian description, the analysis is completely analogous to the one presented above for the intermediate and large friction regime. It yields a cusp pointing upwards with an exponent $2 \beta_{1}-v z=0.37$. This cusp structure necessarily leads to a minimum in the Arrhenius-plot at a temperature $T^{*}>T_{\mathrm{c}}$, which is in accordance with the findings in ref. [5].

We thank H. WAGNER for valuable discussions and a critical reading of the manuscript.
W

\section{REFERENCES}

[1] Hedvall J. A., Hedin R. and Persson O., Z. Phys. Chem. B, 27 (1934) 196.

[2] For a review of the experimental work see Voonhoeve R. J. H., A.I.P. Conf. Proc., 18 (1973) 19; some recent experiments are ref. $[3,4,6-10]$.

[3] Dresselhaus G., Howard I. A., Wassermann B., Dresselhaus M. S. and Zeiger H. J., in Many-Body Phenomena at Surfaces, edited by D. LANGRETH and H. SuHL (Academic, London) 1984, p. 565.

[4] Mehta R. S., Dresselhaus M. S., Dresselhaus G. and Zeiger H. J., Phys. Rev. Lett., 43 (1979) 970.

[5] Measor J. C. and Afzulpurkar K. K., Philos. Mag., 10 (1964) 817.

[6] Sales B. C. and Maple M. B., Phys. Rev. Lett., 39 (1977) 1636.

[7] Sales B. C., Maple M. B. and Vernon F. L., Phys. Rev. B, 18 (1978) 486.

[8] Sales B. C., Cabrera A. L. and Maple M. B., Solid State Commun., 30 (1979) 119.

[9] Sales B. C., Turner J. E. and Maple M. B., Phys. Rev. Lett., 44 (1980) 586.

[10] Shanaberger M. R., Phys. Rev. Lett., 43 (1979) 1964.

[11] SuHL H., Phys. Rev. B, 11 (1975) 2011.

[12] SuHL H., in The Physical Basis for Heterogeneous Catalysis, edited by E. Draugus and R. JAFFEE (Plenum Press, New York, N. Y.) 1975, p. 427.

[13] D'Agliano E. G., Schaich W. L., Kumar P. and SuHL H., in Nobel Symposium 24, Collective Properties of Physical Systems, edited by B. and S. LUNDQUIST (Academic Press, New York, N. Y.) 1973 , p. 200.

[14] D'Agliano E. G., Kumar P., Schaich W. and Suhl H., Phys. Rev. B, 11 (1975) 2122.

[15] Kramers H. A., Physica, 7 (1940) 284.

[16] Grote R. F. and Hynes J. T., J. Chem. Phys., 73 (1980) 2715.

[17] Hängi P. and Mojtabai F., Phys. Rev. A, 26 (1982) 1168.

[18] Carmeli B. and Nitzan A., Phys. Rev. A, 29 (1984) 1481.

[19] Diehl H. W. and Dietrich S., Z. Phys. B, 51 (1983) 343; Erratum B, 52 (1983) 171.

[20] Kinzelbach H., Diploma Thesis, Universität München (1986), unpublished.

[21] Dietrich S. and Kinzelbach H., to be published.

[22] Borman V. D., Prvovarov A. N. and Troyan V. I., Pis'ma Ž. Ėksp. Teor. Fiz., 39 (1984) 458 (English translation: Sov. Phys. JETP Lett., 39 (1984) 556).

[23] DieHL H. W. and Dietrich S., Z. Phys. B, 42 (1981) 65; 50 (1983) 117.

[24] Амтт D. J., Field Theory the Renormalization Group and Critical Phenomena (2nd rev. ed., World Scientific) 1984, p. 294.

[25] Dengler R., Iro H. and Schwabl F., Phys. Lett. A, 111 (1985) 121.

[26] Dietrich S. and DieHL H. W., Z. Phys. B, 43 (1981) 315.

[27] Weller D., Alvarado S. F., Gudat W., Schröder K. and Campagna M., Phys. Rev. Lett., 54 (1985) 1555. 